\title{
Magnetically Controlled Growing Rods: The Experience of Mechanical Failure from a Single Center Consecutive Series of 28 Children with a Minimum Follow-up of 2 Years
}

\author{
Alastair Beaven, Adrian C. Gardner, David S. Marks, \\ Jwalant S. Mehta, Matthew Newton-Ede, Jonathan B. Spilsbury \\ Spinal Deformity Service, The Royal Orthopaedic Hospital, Birmingham, UK
}

Study Design: Retrospective observational study of a continuous series of 28 children.

Purpose: To determine the mechanical failure rate in our cohort of children treated with magnetically controlled growth rods (MCGRs). Overview of Literature: Previous studies report a MCGR mechanical failure rate of $0 \%-75 \%$.

Methods: All patients with MCGR implantation between 2012 and 2015 were examined and followed up for a minimum of 2 years. A retrospective evaluation of contemporaneously documented clinical findings was conducted, and radiographs were retrospectively examined for mechanical failure. The external remote controller (ERC)-specified length achieved in the clinic was compared to the length measured on subsequent radiographs.

Results: Fourteen mechanical failures were identified in 28 children (50\%) across a total of 52 rods (24 pairs and four single constructs). Mechanical failures were due to: failure to lengthen under general anesthesia (seven children), actuator pin fracture (four), rod fracture (one), foundation screw failure (one), and ran out of rod length (one). Of the 14 mechanical failures, six were treated with final fusion operations (reflecting limited further growth potential), and eight patients were treated with the intention for further lengthening. We therefore consider these eight patients to represent the true incidence of mechanical failure in our cohort (29\%). The difference between the ERC length and radiographic length was found to be identical in $11 \%$ cases; $35 \%$ were overestimates, and $54 \%$ were underestimates. The median underestimate was $2.45 \mathrm{~mm}$ whereas the median overestimate was $3.1 \mathrm{~mm}$ per distraction episode. In total, $95 \%$ of all ERC distractions were within $\pm 10 \mathrm{~mm}$ of the radiographic length achieved over a median of nine distraction episodes.

Conclusions: Our series is the most comprehensive MCGR series published to date, and we present a mechanical failure rate of 29\%. Clinicians should be mindful of the discrepancies between ERC length and radiographic measurements of rod length; other modalities may be more helpful in this regard.

Keywords: Scoliosis; Spine; Complication

Received Mar 18, 2018; Revised Jul 8, 2018; Accepted Jul 25, 2018

Corresponding author: Alastair Beaven

Spinal Deformity Service, The Royal Orthopaedic Hospital, The Woodlands, Bristol Road South, Northfield , Birmingham, West Midlands, B31 2AP, UK

Tel: +44-7912-860-775, Fax: +44-1216-854-264, E-mail: Alastairbeaven@traumaresearch.uk 


\section{Introduction}

\section{Background}

Operative treatments for early-onset scoliosis include the insertion of spinal instrumentation to prevent curve progression and affect curve correction through serial lengthening. One such system is the magnetically controlled growth rod system (MCGR; NuVasive Specialized Orthopedic Inc., San Diego, CA, USA). The MCGR system comprises an implantable rod, the external remote controller (ERC), and accessories. The implanted MCGR is used to prevent the progression of the scoliosis while allowing continued spinal growth. After surgical implantation, the ERC is placed externally over the patient's spine at the location of the actuator in the MCGR to increase the length of the construct through periodic, non-invasive distraction of the rod in the conscious child. The National Institution for Health and Care Excellence (NICE) published a case for the recommendation of MCGR in 2014 [1]. They stated that the MGCR system should be considered for use in children aged $\geq 2$ years with scoliosis who require surgery to correct their spinal curvature. NICE concluded that the MCGR system demonstrated cost savings compared to conventional rods, largely because it prevents repeated invasive surgical procedures.

Since the publication of the NICE recommendation, there have been examples of MCGR failures discussed at a consensus meeting [2] and in the literature [3-9]. There have also been analyses of explanted rods [10-12]. Discussions regarding the failure of MCGRs have attempted to determine a mechanical failure rate but have used data that were compiled from multiple centers, conducted with few cases, or followed up for only a short time.

\section{Outline}

We report a continuous series of MCGR patient data from a UK specialist regional spinal deformity service for over 4.5 years that comprised 28 patients with a minimum follow-up of 2 years. This is the largest published continuous series of MCGRs from a single center with a minimum follow-up of 24 months. Children with early-onset scoliosis at the Royal Orthopaedic Hospital, Birmingham are offered corrective surgery after considering the following conventional criteria: (1) degree and extent of curve; (2) spinal maturity; (3) location of curve; and (4) potential for progression. Our center uses MCGRs if at all possible for all indications according to national guidance and has no absolute contraindications for MCGR insertion. Exceptions are made when other clinical issues cause relative contraindications, such as being unable to undergo regular computed tomography. After instrumentation with the MCGR, the children are followed up through a dedicated lengthening clinic at 3-month intervals; they undergo lengthening of approximately $10 \mathrm{~mm}$ each year.

The ERC is used to lengthen the MCGR in the conscious child, and subsequent radiographs are taken to document lengthening and to screen for complications (failure to lengthen, broken rods, incorrectly positioned rods, worsening deformity). To limit exposure to ionizing radiation, radiographs are performed after every two to three ERC lengthening episodes in the clinic-the equivalent of two radiographs per year-mirroring the practice in traditional growing rods (TGRs).

The primary aim of the present study was to assess the mechanical failure rate of MCGRs in our cohort. Furthermore, to understand why some rods failed to lengthen, the secondary aim was to evaluate the degree of lengthening specified by the ERC in the clinic against the lengthening measured on a plain film radiograph.

\section{Materials and Methods}

\section{Recruitment}

All children who underwent implantation of MCGRs at a single spinal deformity service between February 2012 and November 2015 were enrolled in this retrospective observational study. The inclusion criteria included the insertion of MCGRs between February 2012 and November 2015 in our center. All children were included, and there were no exclusions.

\section{Data collection}

Institutional review board approval was granted for this study (internal audit no., 16-040). Contemporaneous data on ERC length were recorded during the clinic lengthening procedures. These data were accessed retrospectively to determine the ERC specified length. Radiographs were accessed retrospectively, and the difference in length of the actuator mechanism was measured. Electronic and paper records were consulted to determine the age, gen- 
der, diagnosis, Cobb angle before rod implantation, date of implantation, rod orientation, rod diameter, and levels of spinal instrumentation [13]. Data were also collected on the failure of devices and the subsequent operative management.

\section{Failure}

Mechanical failure was defined as (1) any rod that the ERC device could not lengthen in an anesthetized child; (2) radiographic evidence of broken metalwork (any part of the MCGR); (3) radiographic evidence of foundation screw failure; and (4) evidence of deep infection requiring revision surgery.

\section{Radiographic evaluation of rod lengthening}

A surgeon who was not associated with the primary surgery or subsequent lengthening episodes measured the radiographs. The radiographs were viewed on the Centricity picture archiving and communication system (PACS; GE Medical Systems Ltd., Buckinghamshire, UK). The rods were reviewed with maximum magnification and on a window level were designed to adequately illustrate the rod-lengthening aperture. Measurements were taken from a line drawn parallel to the actuator pin in the rod-

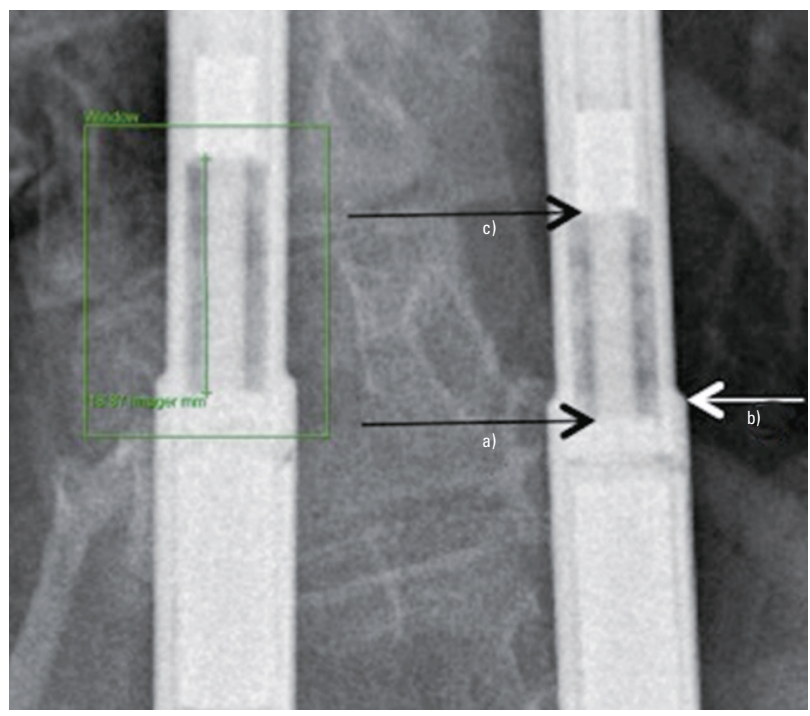

Fig. 1. Method of measuring rod length. Region of Interest Window to ensure adequate exposure of the radiographic area of interest. ${ }^{\text {al Bottom }}$

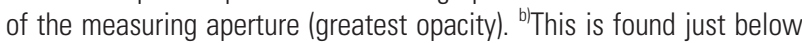
the rod collar. ${ }^{c /}$ Measure to top of the measuring aperture (greatest opacity). Express length in millimeters to nearest $0.1 \mathrm{~mm}$, i.e., $18.9 \mathrm{~mm}$ in this figure. lengthening aperture using the PACS software standard measurement system. Care was taken to determine the superior and inferior margins of the measurement window using the areas of greatest opacity (Fig. 1).

\section{Calibration}

Radiographs in this cohort were not routinely calibrated, and the projections and exposures may vary between different radiographs. The rod diameter was measured on each radiograph using the same measurement software function on PACS. Comparing this measurement against a known rod diameter was used as a calibration technique.

\section{Data analysis}

The data were analyzed retrospectively in Microsoft Excel (for Mac 2011 ver. 14.6.5; Microsoft Corp., Redmond, WA, USA). Nonparametric data are presented as the median (interquartile range [IQR]). Inter- and intrarater variability was assessed via Bland-Altman to determine the mean agreement and limits of agreement. To test both inter- and intrarater variability, ten radiographs were randomly selected for additional measurement by the original researcher and a second senior researcher.

\section{Exclusions}

All failed rods were excluded from the length comparison after the point that failure was identified.

\section{Results}

\section{Demographics}

Twenty-eight children underwent MCGR system lengthening using a total of 52 rods between February 2012 and November 2015 (24 paired rod configurations and four single rod constructs). The median age at implantation was 6 years and 10 months (IQR, 5 years and 2 months to 9 years and 5 months). The children had the following diagnoses: unknown syndrome (nine patients); idiopathic scoliosis (eight); known syndrome (eight); congenital (two); and neuromuscular disorder (one). The most common cephalic level was T2 (13/28 patients), and the most common caudal level was L3 (10/28 patients). The median follow-up length was 3 years and 4 months (IQR, 2 years 
and 6 months to 4 years and 4 months); the minimum follow-up was 2 years and 2 months. The complete demographic data are presented in Table 1.

\section{Calibration}

To calibrate the radiographs, all rod diameters were measured and compared against the known rod diameter of the implanted rod. The difference was $0.1 \mathrm{~mm}$ (IQR, $0.1-0.2 \mathrm{~mm}$ ). The limits of agreement for interobserver error were $0.5 \mathrm{~mm}$ ( $95 \%$ confidence interval $<5 \mathrm{~mm}$ ). The
Table 1. Demographics of patients with MCGR rods in this cohort $(n=28)$

\begin{tabular}{lc} 
Patients with MCGR & Value \\
Male & 9 \\
Female & 19 \\
\hline Age & 7 yr 1 mo (61-129 mo) \\
Cobb angle $\left(^{\circ}\right)$ & $61.5(45-73)$ \\
\hline Rods inserted & 52 \\
\hline Clinic lengthening episodes per patient & $9(6-12)$ \\
Radiographs per patient & $4(3-5.5)$ \\
\hline
\end{tabular}

Values are presented as number or median (interquartile range). MCGR, magnetically controlled growth rod.

Table 2. Outcomes of all rods $(n=28)$

\begin{tabular}{|c|c|c|c|}
\hline Failure & Outcome & Time to failure (yr, mo) & Age at failure (yr, mo) \\
\hline $\mathrm{N}$ & Continue to lengthen & NA & NA \\
\hline N & Continue to lengthen & NA & NA \\
\hline N & Continue to lengthen & NA & NA \\
\hline N & Continue to lengthen & NA & NA \\
\hline N & Continue to lengthen & NA & NA \\
\hline N & Continue to lengthen & NA & NA \\
\hline N & Continue to lengthen & NA & NA \\
\hline N & Continue to lengthen & NA & NA \\
\hline N & Continue to lengthen & NA & NA \\
\hline $\mathrm{N}$ & Continue to lengthen & NA & NA \\
\hline $\mathrm{N}$ : fail to lengthen in clinic & Lengthened successfully under GA & NA & NA \\
\hline $\mathrm{N}$ : fail to lengthen in clinic & Lengthened successfully under GA & NA & NA \\
\hline Y: fail to lengthen under GA & Final fusion & 1,3 & 6,10 \\
\hline Y: fail to lengthen under GA & Plan for final fusion & 2,0 & 13,1 \\
\hline Y: fail to lengthen under GA & Final fusion & 2,3 & 11,7 \\
\hline Y: fail to lengthen under $\mathrm{GA}$ & Plan to exchange for further MCGR & 3,1 & 7,6 \\
\hline Y: fail to lengthen under GA & Converted to traditional rod & 1,3 & 12,0 \\
\hline Y: fail to lengthen under GA & Rods explanted and braced ${ }^{\text {a) }}$ & 1,3 & 7,3 \\
\hline Y: fail to lengthen under GA & Final fusion & $1 / 6$ & 12,11 \\
\hline Y: broken rod & Revised to further $\mathrm{MCGR}^{\mathrm{b})}$ & $1 / 6$ & 7,1 \\
\hline Y: ran out of rod length & Rods exchanged & 2,3 & 6,8 \\
\hline Y: broken actuator pin & Plan to explant & 3,8 & 7,8 \\
\hline Y: broken actuator pin & Final fusion & 5,4 & 14,5 \\
\hline Y: broken actuator pin & Final fusion ${ }^{c)}$ & 1,8 & 8,0 \\
\hline Y: broken actuator pin & Plan for final fusion & 1,3 & 12,2 \\
\hline Y: screws pulling out & Final fusion & 2,7 & 10,0 \\
\hline ?: fail to lengthen in clinic & Plan for lengthening under GA & 2,3 & 6,8 \\
\hline ?: fail to lengthen in clinic & Plan for lengthening under GA & 4,0 & 9,1 \\
\hline
\end{tabular}

NA, not applicable; GA, general anesthesia; MCGR, magnetically controlled growth rod.

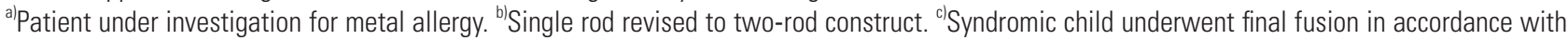
family's wishes. 
limits of agreement for intra-observer error were $0.25 \mathrm{~mm}$ (95\% confidence interval $<2.5 \mathrm{~mm}$ ).

\section{Failure}

Device failure occurred in 14 children (50\%), leading to a decision for operative revision in all 14 cases, with ten operations performed and four operations planned. Of the operations performed, six were final fusion operations, one involved exchange for TGRs, one involved explantation, and two involved exchange for further MCGR systems.

Final fusion operations were performed if the children were reaching skeletal maturity or if further growing potential was limited because of underlying syndromes. Exchange for TGRs was performed as a lengthened schedule was more acceptable (every 6 months under general anesthesia [GA] rather than every 3 months awake). Explantation was performed at the patient's request, and subsequent management was performed by bracing only. One revision to further MCGRs was performed because the rod had run out of length, and more growing was desired. The other MCGR revision involved transferring from a one-rod construct to a two-rod construct after rod fracture.

Of the four operations planned, two are final fusion, one involves explantation, and one involves exchange with another MCGR system. The identified shortcomings in the 14 patients were as follows: failure to lengthen under GA (seven children), actuator pin fracture (four children), rod fracture (one child), foundation screw failure (one child), and ran out of rod length (one child). One child was noted to have deep infection during rod explantation. All failures and outcomes are presented in Table 2.

The median time from implantation to the identification of device failure was 2 years (IQR, 1 year and 3 months to 2 years and 3 months). The median age of the children at identification of failure was 11 years and 7 months (IQR, 7 years and 7 months to 13 years and 0 months).

\section{Clinic lengthening}

There were 235 episodes of clinic lengthening in 28 children. There was a median of nine lengthening episodes per child (IQR, 6-12). The median total ERC length over the time period observed was $19.4 \mathrm{~mm}$ per rod (IQR, $10.8-25.1 \mathrm{~mm})$.

\section{Radiographic measurement}

There were 121 radiographs performed in the 28 children. There was a median of four radiographs per patient (IQR, $3-5.5)$. The median total radiographic length over the time period observed was $19.7 \mathrm{~mm}$ per rod (IQR, 15.3$24.7 \mathrm{~mm})$.

\section{External remote controller versus radiographic mea- surements}

The ERC length was the same as the radiographic length in $11 \%$ of cases whereas it was overestimated in $54 \%$ of cases and underestimated in 35\%. The median underestimate was $2.5 \mathrm{~mm}$, and the median overestimate was 3.1 $\mathrm{mm}$ per distraction episode. In total, $95 \%$ of all ERC distractions were within $\pm 10 \mathrm{~mm}$ of the radiographic length achieved over a median of nine distraction episodes.

\section{Discussion}

\section{Failures}

Our data have not identified a specific reason for device failure. Failure was not associated with the degree of curve, length of time, or age of the child. The overall mechanical failure rate (all causes) in our cohort was 50\%, which agrees with the failure rate of TGR systems that has been previously reported (approximately $40 \%-70 \%$ ) [3,14-17]. The patient's size, contouring of rods, actuator magnets that are too close to one another, and actuator magnets too close to the apex of the curve are factors associated with the failure of magnetically controlled lengthening devices [18].

The fact that reported failure rates for growing constructs (both conventional and MCGR) in scoliosis are so variable makes it difficult to compare between different centers. Differing methodologies, endpoints, and length of follow-up account for some of the differing rates, notwithstanding alternative surgical techniques and lengthening strategies. The failure rate observed in our cohort is received favorably compared to TGRs because MCGR systems require fewer surgeries than conventional growing rod systems and therefore generate fewer non-mechanical complications. 


\section{Actuator pin fracture}

Our series revealed four actuator pin fractures in 28 children (14\%). Of these four pin fractures, two children were treated with spinal fusion, and a third is planned to undergo fusion. Fusion was chosen because it was felt that they had no more growing potential, which lends weight to a theory of Joyce et al. [11] that pin fracture is an effect rather than a cause. If these spines were coming to the end of their natural growth, then the lack of biomechanical compliance in further lengthening may manifest itself as actuator pin fracture. The fourth child with a fractured actuator pin is scheduled for rod exchange with a further MCGR system.

\section{Failure to lengthen under general anesthesia}

Seven children had MCGRs that failed to lengthen under GA. Two of these children (age, 13 and 14 years) underwent fusion because there was limited potential for further lengthening. The other four children underwent rod exchange, either for further MCGR systems (two children) or TGR systems (two children). Cheung et al. [18] reported that increased body habitus and a reduced distance between internal magnets significantly influence rod slippage events. These factors were not examined in our study.

\section{Other failures}

One child had a rod fracture, one rod ran out of length for further lengthening, and one child's screws cut out. Two of these children were treated with exchange and one underwent fusion.

\section{Failure summary}

Overall, 14 children (50\%) experienced MCGR system failure. Six of these children were treated with final fusion operations, which suggests that the MCGR 'failure' occurred in the final stages of spinal growth. Seven children underwent MCGR exchange after their observed failure; therefore, we believe a true mechanical failure rate of $26 \%$ to be a more accurate representation of our experience with MCGRs. The literature has not yet determined what makes rods fail or even what constitutes rod failure. While a rod fracture or foundation screw cut out is a clear construct failure, true MCGR system failure can be more difficult to define. These mechanical devices may have simply reached the end of their useful role with regards to spinal growth. If the native spine has nothing left to give, then it is futile to expect an MCGR to offer any more growth. In these cases, the mechanisms may 'fail', either by fracture of actuator pin or by internal mechanism failure that is not radiologically evident. We distinguish failure in which a further MCGR system is inserted from failure that is treated by final spinal fusion. The exact conditions for failure may become more obvious over time, and there certainly appears to be some correlation in the literature with increasing body habitus, age of the child, and orientation of magnets $[14,18]$. However, we are hesitant to label cases as failures if the children subsequently went on to be treated with final spinal fusion, which would be appropriate for the child in any event.

\section{Comparison with published literature}

To the best of our knowledge, we present the largest continuous series of MCGR patients from a single specialist center with a minimum follow-up of 2 years. Our data are similar to previously published studies regarding the age of child at implantation, degree of curve, and length of follow-up. Previous studies report a MCGR mechanical failure rate (all causes) of $0 \%-75 \%[3,19,20]$, and there have been two specific actuator pin failures published as a case report [21]. In their revised quantitative analysis of four papers (one of which was unpublished) [4,22,23], the NICE external assessment center reported five MCGR device failures in 80 patients (6\%).

Since the NICE report, various case series of MCGRs have been published. We were interested in series that had a comparable number of cases or a similar minimum follow-up as our cohort. We identified seven papers of similar demographics that reported mechanical failure rates of $12 \%-50 \%$.

Dannawi et al. [4] reported four cases of failure in 34 children (12\%). Their failures included two children who failed to distract and two with rod breakages. Their series was from a single center but only had a minimum followup of 12 months. Hickey et al. [5] reported a case series of eight children who were followed up for a minimum of 23 months. They found failure in four children (50\%) due to rod breakage in one, foundation screw failure in one, and failure of distraction in two. Choi et al. [3] demonstrated a complication rate of $28 \%$ from a multicenter 
study with a mean follow-up of 19 months. Complications included broken rods, loss of lengthening, fixationrelated failure, and infections. Akbarnia et al. [6] reported on four children $(33 \%)$ with unplanned revision surgeries in a series of 12 MCGR patients with a mean follow-up of 2.5 years. Hosseini et al. [7] reported that 10 of 23 patients (44\%) with a 2-year follow-up from 15 different sites had implant-related mechanical complications. These complications included pedicle screws pulling out, rod fracture, pain on distraction of one rod, loss of distraction, lack of lengthening with intended distraction, and rods shortened instead of lengthened. No rate was mentioned for the above complications. Kwan et al. [8] reported an unplanned re-operation rate of $46.7 \%$. Their data were collected from 30 patients from six institutions. The included reasons for unplanned re-operation were as follows: failure of rod distraction (six cases), proximal foundation failure (four cases), rod breakage (two cases), infection (two cases), and coronal imbalance (one case). Lebon et al. [9] described a series of 30 patients with MCGR implantation from seven separate European centers. Their mechanical failure rate of $43 \%$ (13/30 patients; loosening of the proximal spinal implant [five cases], rod breakage [two cases], failure to lengthen [six cases]) is similar to that reported in our cohort.

Multicenter series may be vulnerable to inconsistencies in management or decision-making and therefore may not be representative of the technology. The heterogeneous nature of patients and families recruited from studies that span multiple countries may also contribute to threats in translatability. An advantage of our series is that all surgeries were performed in the same center, by the same surgical team, and in a multi-disciplinary environment with experience with these patients. Therefore, we consider our patient cohort to currently be the most reliable when drawing inferences on mechanical MCGR failure.

Panagiotopoulou et al. [10] have recently theorized that actuator pin fracture is caused by secondary corrosion of the internal mechanism, and debris building up inside the mechanism may be responsible. This is in contrast to Joyce et al. [11], who believe that rod failure is due to natural off-axis loading of the spine, and that actuator pin fracture is an effect of rod failure rather than a cause.

\section{Inconsistencies of rod distraction in clinic}

This study found inconsistencies in the ERC measure- ments compared to the radiographic assessments of length. Clinicians must be mindful that ERC measurements do not necessarily reflect the radiographic change in length and can both overestimate and underestimate lengthening. In this series, $95 \%$ of the discrepancies were in the range of $\pm 10 \mathrm{~mm}$ over an average course of nine distraction episodes. This may not be surprising if one considers the inexact nature of perfectly locating the subcutaneous magnets and the intolerance to lengthening found in some children in clinics. A previous study found that the ratio of true distraction length to intended distraction length is 0.33 ; thus, clinic lengthening overestimates actual lengthening [24].

Evaluating the rod length may be better measured by ultrasound. Measuring by ultrasound can be performed in the clinic simultaneously with measuring lengthening, and it may be a more accurate measure of rod length than the ERC, as has been previously suggested $[25,26]$. However, ultrasound can still overestimate length compared to achieved length as measured by X-rays [27]. Ultrasound has the advantage of being a source of non-ionizing radiation and thus can be performed more frequently than Xrays. More frequent assessments of rod length are likely to determine failure to lengthen more quickly than waiting for radiographs. X-rays must still be used in the management of patients with MCGRs because ultrasound cannot reliably detect actuator pin fracture, but their use can be reserved for cases in which rods do not lengthen under ultrasound or at an acceptable time interval.

The measured lengthening of MCGRs may not be a helpful metric. In our study, 17 children were syndromic; therefore, absolute measurements of length and height may have had limited value. We hypothesized that growth velocity is of more utility, and comparing rod length velocity to growth velocity is likely to be more useful. This represents an avenue for future research.

\section{Limitations}

The measuring of rods by radiographic evaluation can be prone to inconsistencies. The radiographs are not routinely calibrated, and projections and exposures may vary between different radiographs. When measuring by eye, researchers are vulnerable to discrepancies in the exact position of measurements. Given that the median calibration measurements were $0.1 \mathrm{~mm}$ (IQR, 0.1-0.2 mm) and that both inter- and intrarater variability were high, we 
concluded that our results are accurate to within $0.5 \mathrm{~mm}$.

\section{Summary}

Any emergent technology should be evaluated before it becomes ubiquitous. It is helpful for scoliosis centers to share real-world data on MCGRs, particularly the incidence of failure, so that a body of evidence may be used to better inform clinical decision-making and patient choices. Our center has reported every case of mechanical failure we have experienced with a minimum of 2 years of follow-up.

We know that differences exist between the degree of measured ERC lengthening and radiographic evaluations of lengthening. Clinicians should be mindful of this discrepancy, and ultrasonography rather than X-rays may be a better measure of rod length. We suspect that growth velocity is more important than absolute rod length, and more research in this area is likely to be beneficial.

The mechanical failure rate of MCGRs has been found to be lower than historical TGRs in our center, and the ability to lengthen rods without a general anesthetic has been found particularly helpful. No specific reasons for actuator pin fracture or internal MCGR mechanism failure have been identified in our cohort, but recognizing trends is difficult with relatively few cases of failure.

\section{Conclusions}

Our series is the most comprehensive series of MCGRs published to date and reports a mechanical failure rate of $29 \%$. Clinicians should be mindful of the discrepancies between ERC length and radiographic measurements of rod length; other modalities may be more helpful in this regard.

\section{Conflict of Interest}

No potential conflict of interest relevant to this article was reported.

\section{Author Contributions}

$\mathrm{AB}$ and $\mathrm{AG}$ original concept and design. $\mathrm{AB}$ data collection and first draft. All authors manuscript editing.

\section{References}

1. Jenks M, Craig J, Higgins J, et al. The MAGEC system for spinal lengthening in children with scoliosis: a NICE Medical Technology Guidance. Appl Health Econ Health Policy 2014;12:587-99.

2. Cheung JP, Cahill P, Yaszay B, Akbarnia BA, Cheung KM. Special article: update on the magnetically controlled growing rod: tips and pitfalls. J Orthop Surg (Hong Kong) 2015;23:383-90.

3. Choi E, Yaszay B, Mundis G, et al. Implant complications after magnetically controlled growing rods for early onset scoliosis: a multicenter retrospective review. J Pediatr Orthop 2017;37:e588-92.

4. Dannawi Z, Altaf F, Harshavardhana NS, El Sebaie $\mathrm{H}$, Noordeen $\mathrm{H}$. Early results of a remotely-operated magnetic growth rod in early-onset scoliosis. Bone Joint J 2013;95-B:75-80.

5. Hickey BA, Towriss C, Baxter G, et al. Early experience of MAGEC magnetic growing rods in the treatment of early onset scoliosis. Eur Spine J 2014;23 Suppl 1:S61-5.

6. Akbarnia BA, Pawelek JB, Cheung KM, et al. Traditional growing rods versus magnetically controlled growing rods for the surgical treatment of early-onset scoliosis: a case-matched 2-year study. Spine Deform 2014;2:493-7.

7. Hosseini P, Pawelek J, Mundis GM, et al. Magnetically controlled growing rods for early-onset scoliosis: a multicenter study of 23 cases with minimum 2 years follow-up. Spine (Phila Pa 1976) 2016;41:1456-62.

8. Kwan KYH, Alanay A, Yazici M, et al. Unplanned reoperations in magnetically controlled growing rod surgery for early onset scoliosis with a minimum of two-year follow-up. Spine (Phila Pa 1976) 2017;42:E1410-4.

9. Lebon J, Batailler C, Wargny M, et al. Magnetically controlled growing rod in early onset scoliosis: a 30case multicenter study. Eur Spine J 2017;26:1567-76.

10. Panagiotopoulou VC, Tucker SK, Whittaker RK, et al. Analysing a mechanism of failure in retrieved magnetically controlled spinal rods. Eur Spine J 2017;26:1699-710.

11. Joyce TJ, Smith SL, Rushton PRP, Bowey AJ, Gibson MJ. Analysis of explanted magnetically controlled growing rods from seven UK spinal centers. Spine (Phila Pa 1976) 2018;43:E16-22. 
12. Teoh KH, von Ruhland C, Evans SL, et al. Metallosis following implantation of magnetically controlled growing rods in the treatment of scoliosis: a case series. Bone Joint J 2016;98-B:1662-7.

13. Cobb JR. Outline for the study of scoliosis. Instr Course Lect 1948;5:261-75.

14. Shinohara K, Takigawa $\mathrm{T}$, Tanaka $\mathrm{M}$, et al. Implant failure of titanium versus cobalt-chromium growing rods in early-onset scoliosis. Spine (Phila $\mathrm{Pa} 1976$ ) 2016;41:502-7.

15. Liang J, Li S, Xu D, et al. Risk factors for predicting complications associated with growing rod surgery for early-onset scoliosis. Clin Neurol Neurosurg 2015;136:15-9.

16. Bess S, Akbarnia BA, Thompson GH, et al. Complications of growing-rod treatment for early-onset scoliosis: analysis of one hundred and forty patients. J Bone Joint Surg Am 2010;92:2533-43.

17. Sankar WN, Acevedo DC, Skaggs DL. Comparison of complications among growing spinal implants. Spine (Phila Pa 1976) 2010;35:2091-6.

18. Cheung JP, Yiu KK, Samartzis D, Kwan K, Tan BB, Cheung KM. Rod lengthening with the magnetically controlled growing rod: factors influencing rod slippage and reduced gains during distractions. Spine (Phila Pa 1976) 2018;43:E399-405.

19. Yilmaz B, Eksi MS, Isik S, Ozcan-Eksi EE, Toktas ZO, Konya D. Magnetically controlled growing rod in early-onset scoliosis: a minimum of 2-year follow-up. Pediatr Neurosurg 2016;51:292-6.

20. Teoh KH, Winson DM, James SH, et al. Magnetic controlled growing rods for early-onset scoliosis: a 4-year follow-up. Spine J 2016;16(4 Suppl):S34-9.
21. Jones CS, Stokes OM, Patel SB, Clarke AJ, Hutton M. Actuator pin fracture in magnetically controlled growing rods: two cases. Spine J 2016;16:e287-91.

22. Akbarnia BA, Cheung $K$, Noordeen $H$, et al. Next generation of growth-sparing techniques: preliminary clinical results of a magnetically controlled growing rod in 14 patients with early-onset scoliosis. Spine (Phila Pa 1976) 2013;38:665-70.

23. Cheung KM, Cheung JP, Samartzis D, et al. Magnetically controlled growing rods for severe spinal curvature in young children: a prospective case series. Lancet 2012;379:1967-74.

24. Rolton D, Thakar C, Wilson-MacDonald J, Nnadi C. Radiological and clinical assessment of the distraction achieved with remotely expandable growing rods in early onset scoliosis. Eur Spine J 2016;25:3371-6.

25. Stokes OM, O’Donovan EJ, Samartzis D, Bow CH, Luk KD, Cheung KM. Reducing radiation exposure in early-onset scoliosis surgery patients: novel use of ultrasonography to measure lengthening in magnetically-controlled growing rods. Spine J 2014;14:2397404.

26. Yoon WW, Chang AC, Tyler P, Butt S, Raniga S, Noordeen $\mathrm{H}$. The use of ultrasound in comparison to radiography in magnetically controlled growth rod lengthening measurement: a prospective study. Eur Spine J 2015;24:1422-6.

27. Cobanoglu M, Shah SA, Gabos P, et al. Comparison of intended lengthening of magnetically controlled growing rods: ultrasound versus X-ray. J Pediatr Orthop 2017 Oct 9 [Epub]. https://doi.org/10.1097/ BPO.0000000000001072. 